\title{
Scaling in Nonstationary Voltammetry Representations
}

\author{
Costas A. Anastassiou ${ }^{a, b, c}$, Kim H. Parker ${ }^{a}$ and Danny O'Hare ${ }^{a}$ \\ ${ }^{a}$ Department of Bioengineering, Imperial College London, SW7 2AZ London, UK \\ ${ }^{b}$ Corresponding author: Costas A. Anastassiou \\ ${ }^{c}$ Present address: \\ Division of Biology, California Institute of Technology, Pasadena 91125, CA \\ Tel.: +16263958965 \\ Fax: +1626796 8876 \\ Email: costas@caltech.edu
}

\section{SUPPORTING INFORMATION}




\section{Supporting Information}

\subsection{Section A}

Space in the axial and the radial dimension was discretised exponentially, very fine near the electrode surface and coarser in the bulk. The mesh expanded $\Delta s=6 \sqrt{2 \tau_{s w}}$ away from the electrode, in the $z$-axis from $z$ $=0$ while in the $r$-axis from $r=r_{e l}$ (electrode edge), a constraint that from 1D-diffusion theory, predicts errors less than $10^{-4} \%$. The mesh parameters used to calculate the current response were: the elements (triangles) at the electrode surface had arc-size equal to $\Delta s / 1000$ and the geometrical factor $\delta=1.07$ for both directions. Once the parameters were chosen, the Delaunay triangulation algorithm from MATLAB was applied to define the computational mesh seen in Figure 1 (S1).
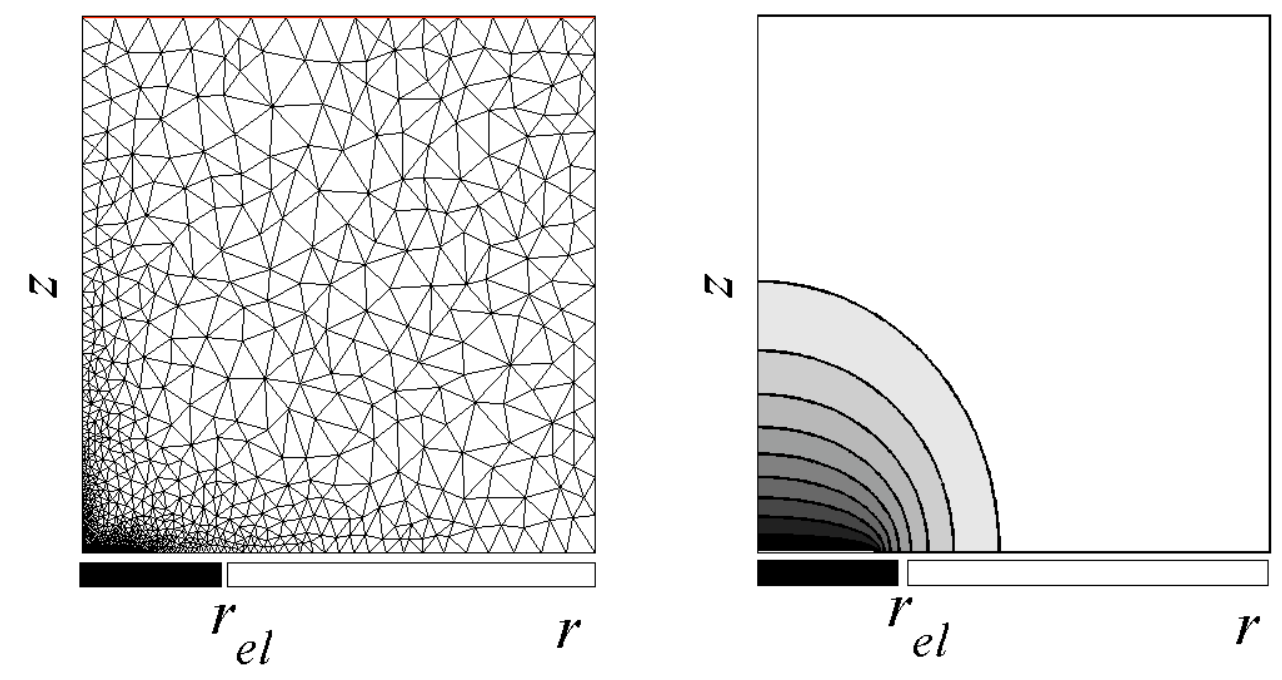

Figure 1: On the left, the computational mesh created by the Delaunay algorithm is shown. The black line below the $r$-axis indicates the position of the electrode whereas the white box the position of the insulation. On the right, the typical hemispherical concentration profile arising from the heterogeneous reaction at the electrode and bulk diffusion is illustreated.

The performance of the computational mesh is illustrated in Fig. 2 and is compared to an accurate semi-analytical solution for the case of completely reversible ET given by Aoki et al. (S2) Aoki's solution includes the edge-effects at the electrode-insulator interface and can thus be used to study phenomena at microelectrodes. In Fig. 2, circles represent the analytical solution whereas solid lines the numerical. The dimensionless parameter $p=\sqrt{\frac{n_{e l} F r_{e l}^{\star} v}{R_{g} T D}}$ where $n_{e l}$ is the number of electron transferred while calculations for three values of $p$ are shown: 1, 5 and 10. As observed, for all cases there is excellent agreement between the analytical and the numerical solution.

Moreover, in order to assess the the accuracy of the algebraic solver UMFPACK with respect to the actual voltammetric dynamics, two types of voltage excitations were applied: $(i)$ for a harmonic chirp of constant 


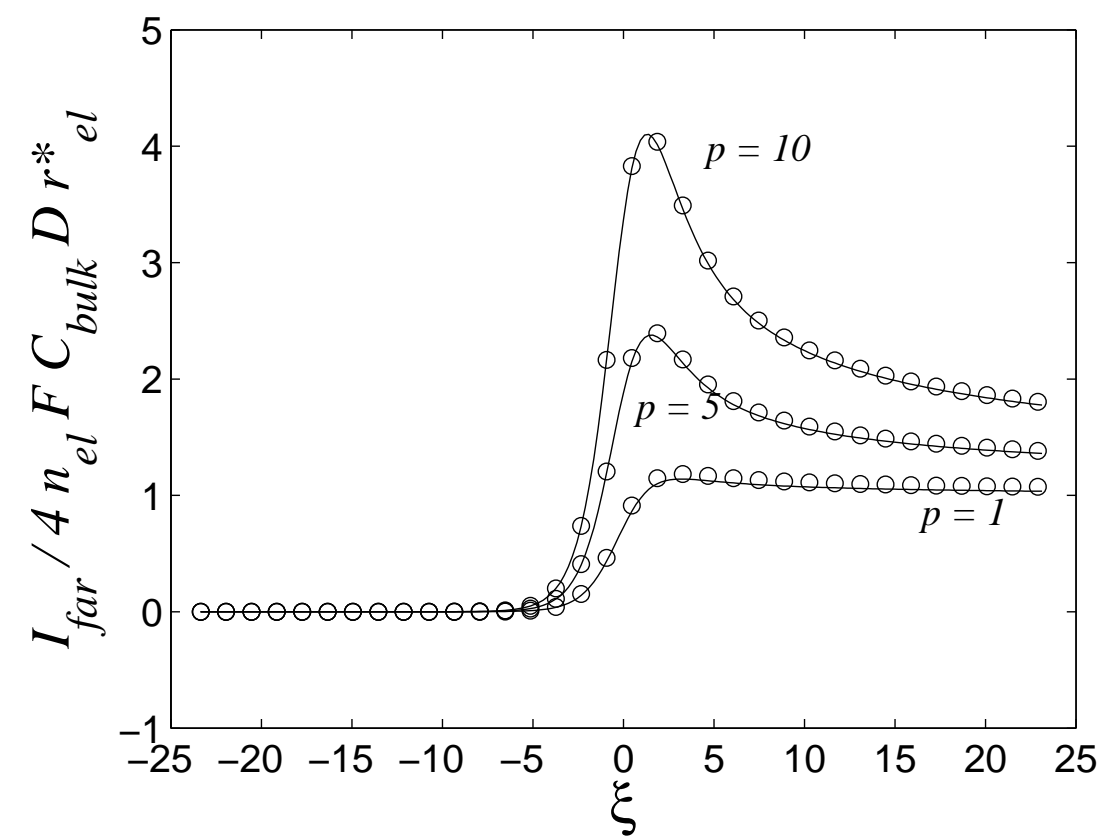

Figure 2: Comparison between the analytical solution given by Aoki et al. (S2) and the numerical solution presented using the computational mesh shown in Fig. 1 with $p=1,5$ and 10.

amplitude $\Delta E^{\star}=0.4 \mathrm{~V}$ and a time-dependent driving frequency $f^{\star}$, and (ii) for ac voltammetry simulations. For both voltage excitations, different meshing parameters were used for an exponentially expanding grid as the one introduced above. For all meshes two cases were tested: a slow reaction $\left(k_{0}=10^{-5} \mathrm{~m} \mathrm{~s}^{-1}\right)$-fast diffusion $\left(D=10^{-9} \mathrm{~m}^{2} \mathrm{~s}^{-1}\right)$ case as well as a fast reaction $\left(k_{0}=10^{-3} \mathrm{~m} \mathrm{~s}^{-1}\right)$-slow diffusion $\left(D=10^{-10}\right.$ $\left.\mathrm{m}^{2} \mathrm{~s}^{-1}\right)$ case. These values explore the limits of $\widehat{t}_{d y n}$ where numerical errors are shown to mainly occur while additional simulations within these two limiting cases were performed to verify the conclusions. For all simulations $\alpha=0.5$.

The frequency $f^{\star}$ of the chirps was consecutively altered every two voltage oscillations in an increasing manner with $f^{\star}=1,25,50,75,100$ and $200 \mathrm{~Hz}$. The mesh cell size $\Delta r=\frac{r_{e l}}{n_{\text {cell }}}$ immediately adjacent to the electrode surface was varied with the number of cells $n_{\text {cell }}=20,100$ and 250 while the mesh geometric growth factor $\delta=1.10$. It was observed that during the frequency modulation, for all cases studied, the relative error remained below $2 \%$. This was also confirmed for much more computationally demanding simulations where $n_{\text {cell }}=500$. On average, the accuracy of the algebraic solver increased by $2 \%$ for a twofold increase in $n_{\text {cell }}$ while for $n_{\text {cell }}=200$ this increase in accuracy was achieved with a 5 -fold increase in CPU-time (S3).

For the ac voltammetry simulations, the voltage excitation parameters were kept constant with $\Delta E^{\star}=$ $0.2 \mathrm{~V}, f^{\star}=200 \mathrm{~Hz}$ and $v=1 \mathrm{~V} \mathrm{~s}^{-1}$ while $n_{\text {cell }}=10,50,100$ and 200 and $\delta=1.07$ and 1.20. In general, it was observed that $50 \leq n_{\text {cell }} \leq 100$ for $\delta=1.07$ resulted in the smallest relative errors, typically $\leq 4 \%$. On the other hand, the CPU usage varied substantially between the different cases as observed in Table 1. The simulations with large $k_{0}$ and small $D$ required nearly double the CPU time than the ones for small $k_{0}$ 
and large $D$. Thus, it is confirmed that the nonlinearity of the heterogeneous reaction introduces significant difficulties to the algebraic solvers. Moreover, the use of very small cell sizes requires increased random access memory which may lead to the solver not converging. For $50 \leq n_{\text {cell }} \leq 100$ and $\delta=1.07$, simulations required less than half the CPU time compared to $n_{\text {cell }}=200$ and $\delta=1.07$. Overall, both analyses suggest that $n_{\text {cell }} \approx 100$ and $\delta=1.07$ offer a good compromise between accuracy and CPU demand and were used for the simulations presented in the manuscript (S3).

\begin{tabular}{|c|c||c|c|}
\hline \multicolumn{2}{|c||}{} & \multicolumn{2}{|c|}{ CPU-time $/ \mathrm{s}$} \\
$n_{\text {cell }}$ & $\delta$ & $k_{0}=10^{-5} \mathrm{~m} \mathrm{~s}^{-1}, D=10^{-9} \mathrm{~m}^{2} \mathrm{~s}^{-1}$ & $k_{0}=10^{-3} \mathrm{~m} \mathrm{~s}^{-1}, D=10^{-10} \mathrm{~m}^{2} \mathrm{~s}^{-1}$ \\
\hline \hline 100 & 1.07 & 3707 & 7368 \\
100 & 1.20 & 2960 & 5175 \\
10 & 1.07 & 1749 & 2647 \\
10 & 1.20 & 1224 & 2010 \\
200 & 1.07 & 7041 & did not converge \\
200 & 1.20 & 4573 & 9849 \\
50 & 1.07 & 2321 & 5349 \\
50 & 1.20 & 3454 & 3987 \\
\hline
\end{tabular}

Table 1: CPU usage as a function of the cell size factor $n_{\text {cell }}$ and the geometric factor $\delta$ for both cases: slow reaction-fast diffusion and fast reaction-slow diffusion. 


\subsection{Section B}

The algorithm khilspec numerically calculates the instantaneous amplitude $\mathrm{A}$, the instantaneous phase $\mathrm{P}$ and the instantaneous frequency $\mathrm{W}$ from a time series $\mathrm{X}$ using the MATLAB-defined functions hilbert and unwrap:

function $(H, W, P)=\operatorname{khilspec}(X)$

analytic $=$ hilbert $(X) ; A=\operatorname{abs}($ analytic); theta $=$ angle(analytic);

$\mathrm{P}=$ unwrap(theta);

$\mathrm{w}=\operatorname{csgolay}(\mathrm{P})$;

csgolay is the Savitzky Golay differentiation filter used to calculate the instantaneous frequency W from the instantaneous phase $\mathrm{P}$ and is given by:

function $\mathrm{dx}=\operatorname{csgolay}(\mathrm{P})$

$\mathrm{N}=2 ; \%$ Order of fitting polynomial

$\mathrm{F}=21 \%$ Total number of points with moving window

$\%$ ensure that row vector is used for calculations and not a column

$(r, c)=\operatorname{size}(P)$;

$\%$ Size of vector to analyse in the second dimension $\mathrm{s}=\operatorname{size}(\mathrm{P}, 2)$;

for $\mathrm{n}=(\mathrm{F}+1) / 2: \mathrm{s}-(\mathrm{F}+1) / 2$,

$\%$ First order time derivative

$\mathrm{dx}(\mathrm{n})=\mathrm{g}(:, 2)^{\prime} * \mathrm{P}((\mathrm{n}-(\mathrm{F}+1) / 2+1):(\mathrm{n}+(\mathrm{F}+1) / 2-1))^{\prime} ;$

end

$\mathrm{dx}=(\operatorname{zeros}(1,(\mathrm{~F}+1) / 2), \mathrm{dx}(((\mathrm{F}+1) / 2): \mathrm{s}-((\mathrm{F}+1) / 2)-1), \operatorname{zeros}(1,((\mathrm{~F}+1) / 2))) ;$ 


\subsection{Section $\mathrm{C}$}

To illustrate the effect of the voltage excitation on the spatiotemporal dynamics of an electrochemical system, in Fig. 3 and 4 the simulation results for sinusoidal voltammetry using the system of equations introduced above are shown: for Fig. $3, f^{\star}=100 \mathrm{~Hz}$ and for Fig. $4, f^{\star}=10 \mathrm{~Hz}$. In both simulations $\Delta E^{\star}=0.4$ V. In both cases, $k_{0}=10^{-3}$ and $D=510^{-10} \mathrm{~m}^{2} \mathrm{~s}^{-1}$ while (A) shows the voltage excitation and (B) the faradaic response $i_{f a r}$. (C)-(F) show the concentration profiles in the four instances designated in (A): (C) $\frac{T}{4}$, (D) $\frac{T}{2}$, (E) $\frac{3 T}{4}$ and (F) $T$ with $T$ being the period of the harmonic oscillation while the box in the $r$-axis indicates the position of the electrode. Comparing the two simulations it becomes apparent that although for (A) similar spatiotemporal distributions are observed for both cases (the initial conditions are identical), for $(\mathrm{F})$ the differences have become substantial. As expected, the larger period $T$ for $f^{\star}=10 \mathrm{~Hz}$ results in the concentration gradient extending further from the electrode surface than for $f^{\star}=100 \mathrm{~Hz}$. For $f^{\star}=100$ $\mathrm{Hz}$ two distinctive features arise in $(\mathrm{F})$ : immediately above the electrode and on the side, immediately at the interface with the insulation, two regions of lower concentration are present. Although both regions also appear for $f^{\star}=10 \mathrm{~Hz}$ the concentration gradient, and thus the resulting diffusive flux, is much smaller there. As a result, for $f^{\star}=100 \mathrm{~Hz}$ the edge-effects are expected to have a more significant impact than for $f^{\star}=10$ $\mathrm{Hz}$ because of the top region resulting in an analyte-flux from the electrode towards the bulk that depletes the analyte-concentration immediately above the electrode as well as due to the concentration gradient at the interface with the insulator. It has to be noted though that in the duration of an ac voltammetry experiment many such oscillations occur and since the system is dissipative, these phenomena will be prominent only close to the electrode. Nevertheless, because the current response according to Eq. ?? is an averaged result of the gradients at the electrode interface, it is directly affected by these processes.

\subsection{Supporting Information References}

(S1) Barber, C. B.; Dobkin, D.P.; Huhdanpaa, H.T. ACM Transactions on Mathematical Software, 1996, $22,469-483$.

(S2) Aoki, K.; Akimoto, K.; Tokuda, K.; Matsuda, H.; Osteryoung, J. J. Electroanal. Chem. 1984, 171, 219-230.

(S3) Anastassiou, C. A. Ph.D. thesis, Imperial College London, 2007. 

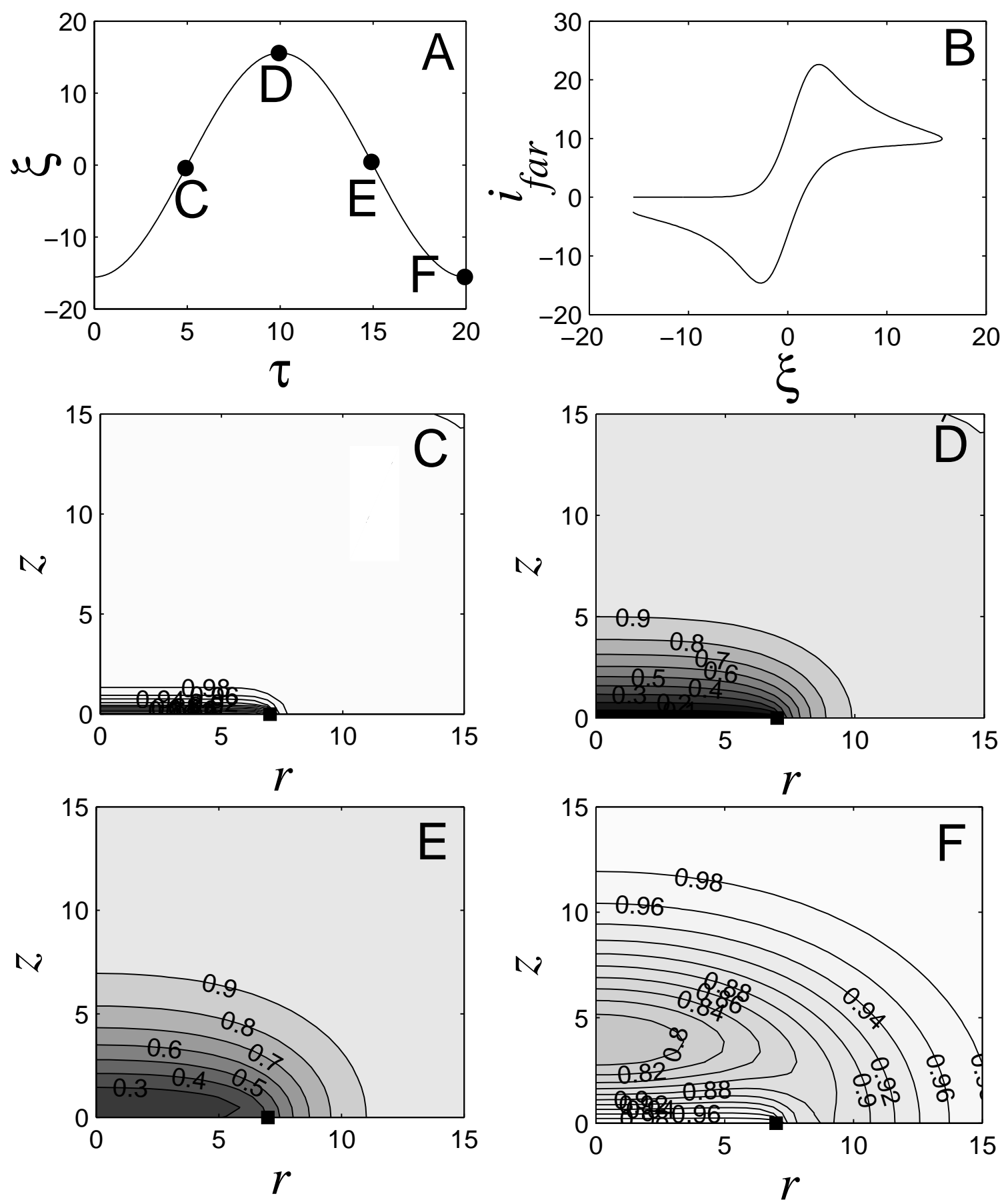

Figure 3: Numerical results for fast sinusoidal voltammetry when parameters are: $\Delta E^{\star}=0.4 \mathrm{~V}$ and $f^{\star}=$ $100 \mathrm{~Hz}$. The physical parameters of the electrochemical are $k_{0}=10^{-3} \mathrm{~m} \mathrm{~s}^{-1}, \alpha=0.5, D=510^{-10} \mathrm{~m}^{2} \mathrm{~s}^{-1}$ while the electrode radius is $r_{e l}^{\star}=3.5 \mu \mathrm{m}$. (A) shows the voltage excitation and (B) the faradaic response $i_{f a r}$. (C)-(F) show the concentration profiles in the four instances designated in (A): (C) $\frac{T}{4}$, (D) $\frac{T}{2}$, (E) $\frac{3 T}{4}$ and (F) $T$ with $T$ being the period of the harmonic oscillation while the box in the $r$-axis indicates the position of the electrode. 

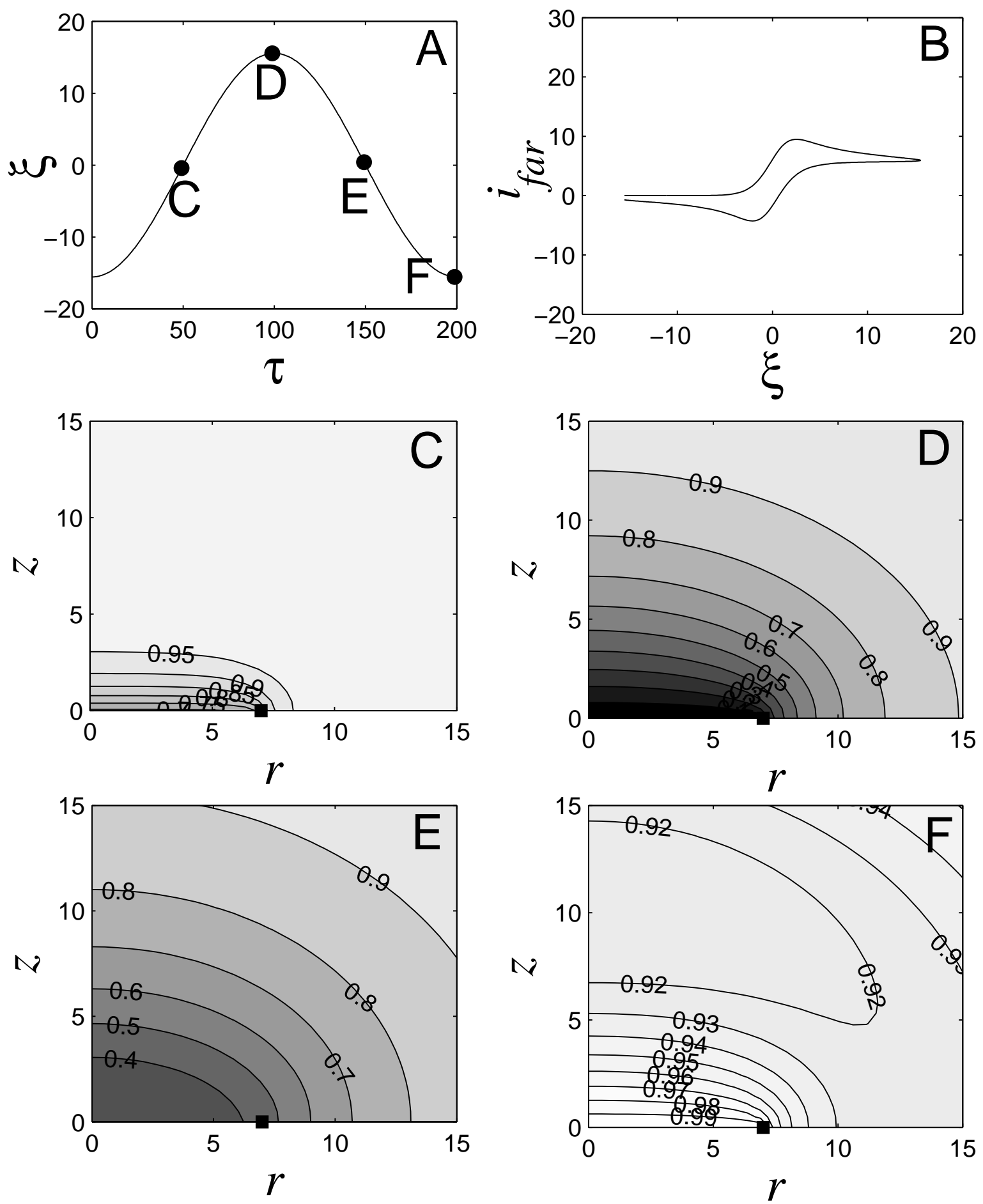

Figure 4: Numerical results for slow sinusoidal voltammetry when parameters are: $\Delta E^{\star}=0.4 \mathrm{~V}, v=0.2$ $\mathrm{V} \mathrm{s}^{-1}$ and $f^{\star}=10 \mathrm{~Hz}$. The physical parameters of the electrochemical are $k_{0}=10^{-3} \mathrm{~m} \mathrm{~s}^{-1}, \alpha=0.5, D=$ $510^{-10} \mathrm{~m}^{2} \mathrm{~s}^{-1}$ while the electrode radius is $r_{e l}^{\star}=3.5 \mu \mathrm{m}$. (A) shows the voltage excitation and (B) the faradaic response $i_{f a r}$. (C)-(F) show the concentration profiles in the four instances designated in (A): (C) $\frac{T}{4}$, (D) $\frac{T}{2}$, (E) $\frac{3 T}{4}$ and (F) $T$ with $T$ being the period of the harmonic oscillation while the box in the $r$-axis indicates the position of the electrode. 